\title{
Proliferative Anterior Optic Membranes in Hydrophobic Acrylic Intraocular Lenses
}

This article was published in the following Dove Press journal:

Clinical Ophthalmology

\author{
Kamran M Riaz $\mathbb{D}^{1}$ \\ Blake L Williams ${ }^{2}$ \\ Steven G Safran ${ }^{3}$ \\ Mark J Gallardo (D) ${ }^{4}$ \\ 'Department of Ophthalmology, Dean \\ McGee Eye Institute/University of \\ Oklahoma, Oklahoma City, OK, USA; \\ ${ }^{2}$ Department of Ophthalmology and \\ Visual Science, University of Chicago \\ Medical Center, Chicago, IL, USA; \\ ${ }^{3}$ Private Practice, Lawrenceville, NJ, USA; \\ ${ }^{4}$ Private Practice, El Paso, TX, USA
}

Purpose: To describe the observed stages of development and management of proliferative anterior optic membranes (AOM) seen on a popularly implanted single-piece hydrophobic intraocular lens (IOL).

Methods: This is an observational series of patients with AOMs managed by three surgeons in different geographical areas in the USA.

Results: AOMs may clinically present in one of the four distinct stages. Most patients were visually asymptomatic, but those with decreased visual acuity (Stage 4) required Nd:YAG laser treatment to remove the AOM. These patients had improved visual acuity without recurrence of the AOM during the one-year follow-up period.

Conclusion: AOMs can present and progress in four distinct stages with variable visual significance. AOMs may be safely and effectively treated with Nd:Yag laser. Occurrence of the membrane may be related to many factors, including high fibronectin content of this IOL. Additional studies are needed to identify incidence, etiology, and best management strategies.

Keywords: anterior optic membrane, hydrophobic intraocular lens, fibronectin, YAG laser

\section{Plain Language Summary}

This observational commentary describes the appearance and management of post-surgical scar tissue (anterior optic membranes; AOMs) that develops on a particular model of intraocular lens in post-cataract surgery patients. We describe four distinct stages of these AOMs as well as offer potential treatment strategies (e.g., laser surgery to remove AOMs) to manage these visually significant postoperative findings. Several potential pathophysiological reasons are also discussed as to why AOMs develop in this particular IOL. We believe that these observations may be of significant interest as this IOL is the preferred choice of many cataract surgeons. We have restricted our commentary to our observations as we believe additional studies are needed to assess the statistical incidence, visually significant pathology and management strategies for this condition. Further studies, ideally with a multinational effort, are needed to better study the incidence, visual significant, and management options (including both surgical and non-surgical treatment strategies) for AOMs.

\section{Background}

This observational commentary describes the appearance and management of postsurgical scar tissue (anterior optic membranes; AOMs) that develops on a particular model of intraocular lens in post-cataract surgery patients. We describe the various stages of this AOM as well as offer potential treatment strategies to manage these visually significant entities. We have restricted our commentary to our observations
Correspondence: Kamran M Riaz Oklahoma, 608 Stanton L Young

Boulevard, Suite 313, Oklahoma City, OK 73104, USA

Tel $+|40527| 1095$

$\mathrm{Fax}+$ I 40527/3680

Email Kamran-Riaz@dmei.org 
as we believe additional studies are needed to assess the statistical incidence, visually significant pathology and management strategies for this condition.

We report observations on a series of patients who underwent uncomplicated cataract surgery with implantation of a single-piece hydrophobic acrylic intraocular lens (IOL) that subsequently developed a membranous growth on the anterior surface of the IOL optic. We have termed this growth as an anterior optic membrane (AOM). AOMs have a similar appearance to epithelial proliferation seen in previous reports with several notable differences. ${ }^{1-3} \mathrm{We}$ will describe similarities and differences of these AOMs as compared to anterior optic proliferations previously mentioned in the literature. Observations of AOMs were made by three surgeons who practice in different geographical and demographic areas of the United States over a period of approximately three years. AOMs were observed in patients who underwent surgery by the study authors as well as patients who underwent surgery elsewhere.

This retrospective observational study was approved by the Institutional Review Board (IRB) of the University of Oklahoma Health Sciences Center (IRB\#: 12,387). The IRB waived the requirement for informed consent for review of medical records as it was determined there was minimal/no risk to patients or residents being studied. This work adhered to the tenets of the Declaration of Helsinki. Patient data confidentiality was maintained throughout the study and manuscript preparation process.

All patients with AOMs had an AcrySof model IOL (Alcon Laboratories, Ft. Worth, TX USA) implanted, including the monofocal (SN60WF and SA60WF models), toric (SN6ATx and SA6ATx models), multifocal (SN6AD1 and SV25T0 models), and combined toricmultifocal style IOL (SND1Tx and SV25Tx models). However, not every patient with this model IOL examined by the authors had an AOM. AOMs were observed both in the early (within 1 month) and late post-operative period (after 3 months) in eyes without significant post-operative inflammation.

Clinically, AOM morphology may present on a spectrum of findings in one of the four distinct stages (Figures 1A-D and 2 and Table 1). We have collectively observed and treated patients with various stage AOMs over the past three years. As this is an observational case series, we cannot report the exact number or percentage of AOMs seen at this time. The majority of AOMs we encountered were visually insignificant (Stage 1-3 $\mathrm{AOM}$ ), though we cannot report the number of patients in each stage. However, some patients developed visual symptoms such as glare, photophobia, and/or a subjective and objective decline in BCVA, typically ranging from 20/ 30 to $20 / 50$ in the affected eye, consistent with stage 4 AOM. All three surgeons have reported these observations to the manufacturer independently.

Patients with stage 4 AOM causing decreased BCVA may benefit from surgical intervention with $\mathrm{Nd}$ :YAG laser treatment (Figure 3). Laser treatment is best applied in a circular, sweeping pattern in the mid-periphery of the anterior optic as previously described, with care given to avoid damage to the IOL. ${ }^{4}$ There have been no recurrences

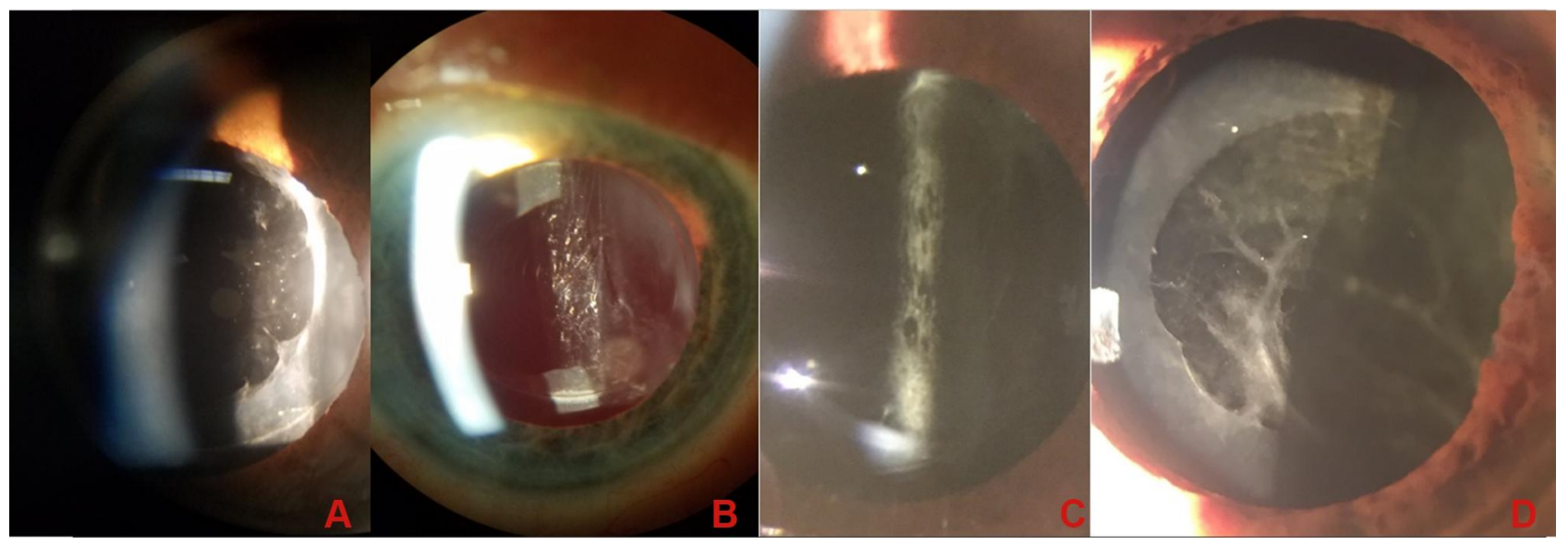

Figure I Stages of anterior optic membrane (AOM) appearance. Stage I AOM (A) features an extension of diaphanous membranous material from the capsulorrhexis edge onto the anterior optic without incursion into the visual axis. Stage 2 AOM (B) is characterized by a slightly denser, cellophane-like membrane with extension into the visual axis. Best corrected visual acuity (BCVA) is minimally affected in Stage I-2. Stage 3 AOM (C) is a denser, partial light-blocking membrane with full involvement of the anterior optic, with or without focal and/or intervening clear spaces. BCVA may be minimally affected. Stage 4 AOM (D) is characterized by an opaque, disorganized fibrotic growth accompanied by moderate-severe loss of BCVA. Stage 3 and 4 AOMs may require YAG laser treatment. 


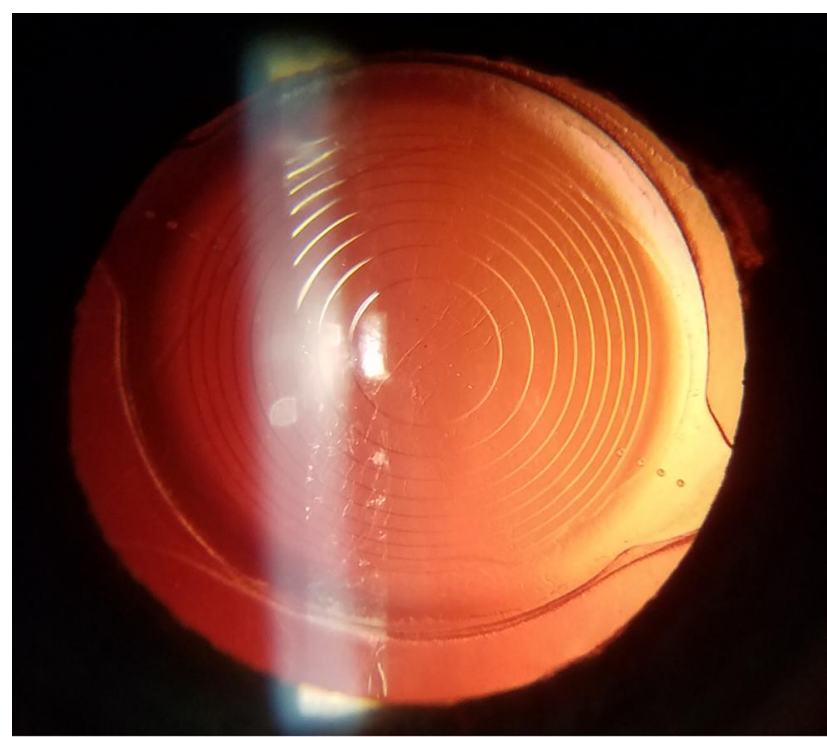

Figure 2 Retro-illumination view of a Stage I AOM.

of AOMs in these eyes with a one-year follow-up period, though it is possible that AOMs may partially or fully recur with longer follow-up. Though we observed improved BCVA in most patients that underwent YAG laser treatment, a formal visual outcomes analysis was not performed as this is beyond the scope of this correspondence.

Reviewing the literature and to the best of our knowledge, we did not encounter any reports concerning incidence of and management of AOMs in the setting of modern phacoemulsification techniques with this model of IOL. There have been reports regarding lens epithelial cell

Table I Classification of Observed Four Stages of Anterior Optic Membranes (AOM)

\begin{tabular}{|l|l|}
\hline $\begin{array}{l}\text { Stage of } \\
\text { AOM }\end{array}$ & Clinical Features \\
\hline Stage I & $\begin{array}{l}\text { Thin translucent layer extending centripetally from the } \\
\text { edge of the capsulorrhexis without involvement of the } \\
\text { visual axis }\end{array}$ \\
\hline Stage 2 & $\begin{array}{l}\text { Denser cellophane membrane with involvement of the } \\
\text { visual axis }\end{array}$ \\
\hline Stage 3 & $\begin{array}{l}\text { Partial light blocking membrane with full involvement } \\
\text { of the anterior optic (with or without intervening } \\
\text { clear spaces) associated with mild decrease in best } \\
\text { corrected visual acuity (BCVA) }\end{array}$ \\
\hline Stage 4 & $\begin{array}{l}\text { Opaque fibrotic membrane with moderate-severe loss } \\
\text { of BCVA }\end{array}$ \\
\hline
\end{tabular}

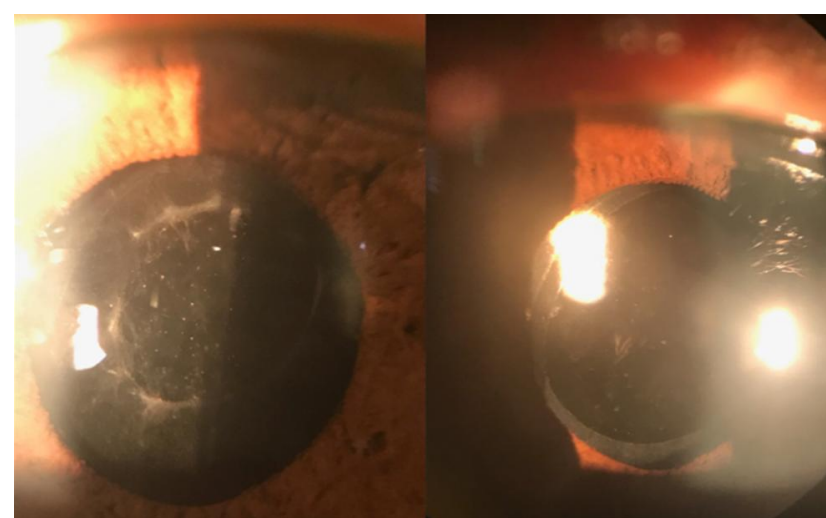

Figure 3 Stage 4 AOM before treatment (left panel) and after YAG treatment (right panel).

(LEC) growth on the anterior surface of IOL optics, but these were on either 3-piece IOLs and/or single-piece polymethyl-methacrylate (PMMA) lenses using older cataract extraction techniques. ${ }^{5,6}$ It is possible that the observed AOMs represent a morphological variant of LEC growth onto the optic. One paper showed a higher incidence of membrane formation in 3-piece acrylic versus silicone and PMMA IOLs and a longer duration to resolution. ${ }^{6}$ AOMs as we have described with this spectrum of stages have not previously been described in the current generation AcrySof IOL models. We suspect that AOMs may occur quite frequently but they are often simply observed as they are usually visually insignificant. Furthermore, we have observed other deposits, opacifications, and calcifications that can occur on IOLs from all manufacturers; AOMs may therefore represent a variant or spectrum of these findings.

The full etiology of AOMs in this IOL platform remains unexplained and is beyond the scope of this commentary, especially since AOMs were seen both in the early and latepostoperative stage over three years. One factor may be related to extracellular matrix proteins, such as fibronectin, vitronectin, and type IV collagen, which have been shown to act as mediators of IOL adhesion to the capsular bag, either directly or through LECs. ${ }^{6,7}$ Specifically, more fibronectin may be bound to the hydrophobic acrylic IOLs than to 2-hydroxyethyl methacrylate (HEMA) containing hydrophilic acrylic IOLs. ${ }^{7}$ We propose that one potential reason for AOM formation may be due to this manufacturer's IOL having a higher fibronectin adherence compared to other IOLs. ${ }^{7-9}$ While higher fibronectin adherence may offer advantages in terms of reducing PCO and IOL rotation, it may also allow for enhanced cell binding and proliferation on the anterior optic of the IOL, resulting in 
AOM formation. Thus, despite their differences, AOMs may develop in a manner that is different from PCO formation. Interestingly, the Sensar IOL (Johnson and Johnson Vision, Jacksonville, FL USA) has similar fibronectin adherence properties to the AcrySof IOL; however, we have not observed AOMs in these IOLs though it is possible other surgeons may have done so. ${ }^{9}$

It is also conceivable that other factors, such as manufacturing technique and processing, may contribute to AOMs. Histological studies may shed further insight on the biochemical composition of AOMs, which may elucidate the pathophysiology present. More research is needed to identify primary and secondary risk factors as well as strategies to prevent AOM formation.

There are limitations to our correspondence. First, because this is an observational study, we must emphasize that no definitive conclusions can be made regarding the etiology and incidence of all four stages of AOMs. Second, it is possible that other surgeons who routinely implanted this IOL have not encountered AOMs or had any poor visual outcomes as a result of AOMs. Third, YAG laser treatment of the anterior IOL is technically challenging and may incur additional, visually significant consequences, such as IOL pitting. Finally, we do not have information regarding lot/batch number of these IOLs to shed light on a manufacturing issue. However, since AOMs were observed by the authors over three years, we maintain that this is not limited to a particular manufactured batch of these IOLs.

In conclusion, we report our collective observations concerning the appearance and management of AOMs in patients with AcrySof IOLs. In addition to describing the four stages of AOMs, we postulate a potential etiology for their development. However, at this time we cannot conclusively point to one causative factor that leads to the development of AOMs in this manufacturer's IOL. While the vast majority of AOMs remain visually insignificant, a small percentage of patients may require surgical intervention as described. Given the popularity of these IOLs, we believe our reported experiences may be of interest to our colleagues. Further studies, ideally with a multinational effort, are needed to better elucidate the incidence, visual significance, and management of our observations.

\section{Presentations}

Preliminary data, images and findings in this manuscript were previously presented at the 2019 American Society of Cataract and Refractive Surgery (ASCRS) Annual Meeting, San Diego, CA, USA.

\section{Author Contributions}

All authors made substantial contributions to conception and design, acquisition of data, or analysis, and interpretation of data; took part in drafting the article or revising it critically for important intellectual content; agreed to submit to the current journal; gave final approval of the version to be published; and agree to be accountable for all aspects of the work.

\section{Disclosure}

None of the authors have any relevant financial disclosures in regards to the subject matter of this presentation. No pharmaceutical company was involved in the conception, development, and/or submission of the data and accompanying manuscript. Kamran M Riaz reports personal fees from Beaver-Visitec, Inc and being a speaker and consultant for Bausch and Lomb, outside the submitted work. The authors report no other potential conflicts of interest for this work.

\section{References}

1. Pande MV, Spalton DJ, Marshall J. In vivo human lens epithelial cell proliferation on the anterior surface of PMMA intraocular lenses. $\mathrm{Br}$ J Ophthalmol. 1996;80:469-474. doi:10.1136/bjo.80.5.469

2. Nagamoto T, Hara E. Postoperative membranous proliferation from the anterior capsulotomy margin onto the intraocular lens optic. J Cataract Refract Surg. 1995;21:208-211. doi:10.1016/S08863350(13)80512-3

3. Turner A, House P. Proliferation of lens epithelial cells on the AcrySof intraocular lens: clinical and histological features of a case. Clin Exp Ophth. 2001;29:426-428. doi:10.1046/j.1442-9071.2001.d01-34.x

4. Vajpayee RB, Angra SK, Honavar SG, Kumar H. Nd:YAG "sweeping" - an indirect technique for clearing intraocular lens deposits. Ophthalmic Surg. 1993;24:489-491.

5. Kurosaka D, Kato K. Membranous proliferation of lens epithelial cells on acrylic, silicone, and poly(methyl methacrylate) lenses. $J$ Cataract Refract Surg. 2001;27(10):1591-1595. doi:10.1016/S0886-3350(01) 00870-7

6. Koch MU, Kalicharan D, van der Want JJL. Lens epithelial cell layer formation related to hydrogel foldable intraocular lenses. $J$ Refract Surg. 1999;25(12):1637-1640. doi:10.1016/S0886-3350(99)00267-9

7. Linnola RJ, Werner L, Pandey SK, Escobar-Gomez M, Znoiko S, Apple DJ. Adhesion of fibronectin, vitronectin, laminin, and collagen type IV to intraocular lens materials in pseudophakic human autopsy eyes. J Cataract Refract Surg. 2000;26(12):1792-1806. doi:10.1016/ S0886-3350(00)00748-3

8. Linnola RJ, Sund M, Ylonen R, Pihlajaniemi T. Adhesion of soluble fibronectin, vitronectin, and collagen type IV to intraocular lens materials. J Cataract Refract Surg. 2003;29(1):146-152. doi:10.1016/ S0886-3350(02)01422-0

9. Ong M, Wang L, Karakelle M. Fibronectin Adhesive Properties of Various Intraocular Lens Materials. Fort Worth, TX, USA: Alcon Laboratories, ARVO; 2013. 


\section{Publish your work in this journal}

Clinical Ophthalmology is an international, peer-reviewed journal covering all subspecialties within ophthalmology. Key topics include: Optometry; Visual science; Pharmacology and drug therapy in eye diseases; Basic Sciences; Primary and Secondary eye care; Patient Safety and Quality of Care Improvements. This journal is indexed on PubMed

Submit your manuscript here: https://www.dovepress.com/clinical-ophthalmology-journal
Central and CAS, and is the official journal of The Society of Clinical Ophthalmology (SCO). The manuscript management system is completely online and includes a very quick and fair peer-review system, which is all easy to use. Visit http://www.dovepress.com/ testimonials.php to read real quotes from published authors. 